\title{
EL PLAN DE TRANSICIÓN Y EGRESO HOSPITALARIO Y SU EFECTO EN EL CUIDADO DE LA SALUD: UNA REVISIÓN INTEGRADA
}

\section{TRANSITION AND DISCHARGE HOSPITAL PLANNING AND ITS EFFECT IN HEALTH CARE: AN INTEGRATED REVIEW}

\author{
Beatriz Sánchez ${ }^{1}$, Gloria Mabel Carrillo², Lucy Barrera ${ }^{3}$
}

${ }^{1}$ Enfermera. Master in Science of Nursing. Profesora Titular Universidad Nacional de Colombia. Facultad de Enfermería. Bogotá, D.C., Colombia, cbsanchezh@unal.edu.co; ${ }^{2}$ Enfermera. Magister en Enfermería. Profesora Asociada Universidad Nacional de Colombia. Facultad de Enfermería, gmcarrillog@unal.edu.co; ${ }^{3}$ Enfermera. Magister en Enfermería. Profesora Titular pensionada. Universidad Nacional de Colombia. Facultad de Enfermería, lbarrerao@unal.edu.co

Rev. U.D.C.A Act. \& Div. Cient. 17(1): 13-23, Enero-Junio, 2014

\section{RESUMEN}

Mediante una revisión integrada de investigación que incluyó búsqueda de ensayos clínicos aleatorizados, estudios descriptivos, estudios de relación, revisiones sistemáticas, metaanálisis y metasíntesis, en bases de datos, como Medline, ScienceDirect, Ovid y Scielo, bajo los descriptores plan de egreso o transición cruzadas con efectividad, cuidado integral, cuidado continuo y cuidado seguro, en el periodo de 1997 a 2013, se exploró la literatura referente a plan de transición y de egreso hospitalario, sus componentes, sus funciones, así como la existencia de evidencia sobre sus efectos en el cuidado de la salud. Como resultado, se encontraron y analizaron 52 referencias clasificadas en cuatro grupos, de acuerdo con su énfasis: 1) Falta de lineamientos claridad e incentivos para adelantar el plan; 2) Desarrollo de instrumentos que respaldan la valoración y medición dentro del plan de egreso; 3) Las características básicas que debe tener un plan de egreso o su desarrollo teórico y, 4) Efectividad del plan de egreso y transición hospitalaria para el cuidado continuo, seguro e integral de los pacientes y sus familias. Con esto, se puede llegar a la conclusión, que existe una literatura variada sobre el plan de transición y egreso hospitalario, que refleja un nivel heterogéneo frente a directrices concretas, para adelantar dichos planes, aunque se recomienda esta gestión en beneficio de los usuarios. La evidencia sobre la efectividad del desarrollo de planes de cuidado, como parte fundamental del cuidado continuo, seguro e integral de los pacientes y sus familias es limitada. Son recomendados estudios de tipo predictivo en este campo.

Palabras clave: Alta del paciente, atención de enfermería, seguridad del paciente.

\section{SUMMARY}

Through an integrated review that included randomized clinical trials search, descriptive studies, association studies, systematic reviews, meta-analyzes and meta-synthesis in databases such as Medline, ScienceDirect, Ovid and Scielo low exit descriptors or Transition Plan cross with Effective, comprehensive Care, and Care continuing, Care insurance in the period 1997-2013, the literature on transition plan and hospital discharge, their components, their functions and the existence of evidence of its effects on health care was explored. As a result, 52 references were found and analyzed, classified into four groups according to their emphasis: 1) Lack of clear guidelines and incentives to advance the retirement plan, 2) Development of tools to support the assessment and measurement within the retirement plan 3) the basic features that should have a retirement plan or its theoretical development and 4) Effective discharge and transition plan for ongoing care hospital, and comprehensive insurance patients and their families. With this, it can be concluded that there is an ample literature on the transition plan and hospital discharge, reflecting a heterogeneous level opposite specific guidelines to put forward these plans, although this management to the benefit of users is recommended. The evidence on the effectiveness of a care plan development as a fundamental part of a continual, assured and comprehensive care of the patients and their families is limited. In this field studies of the predictive type are recommended.

Key words: Patient Discharge, nursing care, patient safety (Decs). 


\section{INTRODUCCIÓN}

Según la Organización Mundial de la Salud (2013), en los últimos años, se ha generado una alarma mundial por el incremento de la enfermedad crónica no trasmisible (ECNT), en especial, en los países en desarrollo. Estas enfermedades son complejas, se prolongan en el tiempo y, por lo general, demandan apoyos de cuidado; quizá por ello, día a día se ha enfatizado más en la necesidad de ofertar a los pacientes un cuidado seguro, continuo e integral (Barrera et al. 2006a; Sánchez, 2011). El Plan de transición y de egreso hospitalario, restringido por algunos al plan de alta hospitalaria, es una expresión de este tipo de cuidado que adquiere relevancia en este contexto y, en particular, cuando en los sistemas de salud, a nivel mundial, el incremento del costo institucional presiona el paso de servicios de mayor a menor complejidad y de la institución al hogar, sin que exista la preparación adecuada para asumir esta responsabilidad de cuidado (Pinto et al. 2006; Barrera et al. 2006b).

El presente trabajo exploró la literatura disponible en los últimos 15 años, tiempo, en el cual, confluyen los mayores cambios en los sistemas de salud y se toma mayor conciencia del incremento de la enfermedad crónica, a nivel mundial.

No se encontraron documentadas búsquedas de esta naturaleza, las cuales, se hacen necesarias para clarificar qué es y cómo se ha abordado el tema de Plan de transición y de egreso hospitalario, sus componentes, sus funciones y la existencia de evidencia sobre sus efectos en el cuidado de la salud.

Se considera un referente para la proyección de enfermería en la generación de propuestas de planes de egreso en las instituciones de salud, la identificación de tópicos de interés investigativo y de indicadores de impacto sobre los que se pueden reportar su efecto en los usuarios y las entidades de salud.

\section{MATERIALES Y MÉTODOS}

A través de una revisión integrada de la investigación (Burns \& Grove, 2012), se identificaron, analizaron y sintetizaron los hallazgos de resultados de estudios independientes sobre plan de egreso y sus componentes.

El procedimiento utilizado para esta revisión fue: 1) Búsqueda y selección de artículo en función de su calidad y relación con el problema elegido; 2) Codificación de cada artículo y elaboración de ficha bibliográfica respectiva; 3) Lectura de las piezas de investigación identificadas; 4) Diseño de una tabla de análisis que incluyó información esencial sobre propósito, método y resultados obtenidos de la investigación; 5) Identificación de hallazgos comunes, comparación y contraste con los resultados prácticos que se han conseguido y,
6) Integración de los resultados obtenidos en los estudios, a través de categorías (Burns \& Grove, 2012).

La estrategia de búsqueda incluyó artículos indexados de ensayos clínicos aleatorizados, estudios descriptivos, estudios de relación, revisiones sistemáticas, metaanálisis y metasíntesis. Las bases de datos usadas fueron: medline, Science Direct, Ovid y Scielo, bajo los descriptores plan de egreso o transición cruzadas con efectividad, cuidado integral, cuidado continuo y cuidado seguro, estableciendo como ventana de observación el periodo comprendido entre 1997 a 2013. La revisión, el posterior análisis y discusión, así como la construcción de las categorías de resultados, se realizaron con base en la experiencia desde la práctica clínica y la docencia en el área de cuidado al paciente crónico y la familia, generando una reflexión crítica sobre los aspectos claves que se deben abordar en las instituciones prestadoras de servicios de salud, para la consolidación de planes de egreso hospitalario.

El grupo investigador estuvo a cargo de la búsqueda, la selección y la revisión de cada una de las piezas de investigación utilizadas. Las categorías obtenidas emergieron de acuerdo a los hallazgos identificados en los estudios; sin embargo, el proceso de selección de la información estuvo orientado teniendo en cuenta la identificación de componentes, las funciones y la existencia de evidencia sobre sus efectos en el cuidado de la salud, respecto a plan de egreso.

\section{RESULTADOS Y DISCUSIÓN}

Se identificaron 252 referencias relacionadas, de las que se revisaron los resúmenes. Después de leerlos, se excluyeron aquellos que no hacían parte del área temática de interés, así como los que estaban duplicados en varias bases de datos. Finalmente, se seleccionaron 52 piezas investigativas, publicadas, que cumplieron con los criterios de inclusión.

Estas 52 publicaciones fueron clasificadas en cuatro categorías, de acuerdo con su énfasis: Falta de lineamientos claridad e incentivos para adelantar el plan, desarrollo de instrumentos, características básicas y desarrollo teórico, efectividad del plan de egreso y transición hospitalaria para el cuidado continuo, seguro e integral de los pacientes y sus familias.

Con relación a Falta de lineamientos, claridad e incentivos, esta categoría incluyó 11 publicaciones, que señalan que, a pesar de estar recomendado realizar plan de egreso hospitalario, se requiere dar claridad e incentivar el desarrollo del mismo. Estos estudios aceptan la importancia y la pertinencia de los planes de transición y de egreso, como elemento fundamental en el cuidado; sin embargo, reflejan falta claridad para desarrollarlos (Cuadro 1). 
Cuadro 1. Estudios que evidencian la falta de claridad e incentivos para realizar un plan de egreso.

\begin{tabular}{|c|c|c|}
\hline Autores/año & Objetivo/Propósito & Resultados \\
\hline $\begin{array}{l}\text { Lin et al. } \\
(2009)\end{array}$ & $\begin{array}{l}\text { Revisar cuáles son los factores que rodean al proceso } \\
\text { de egreso en una unidad de cuidado intensivo. }\end{array}$ & $\begin{array}{l}\text { Pocas unidades tienen descrito cómo adelantar dicho plan. La } \\
\text { ausencia de una clara directriz, se asocia con mayor mortali- } \\
\text { dad. }\end{array}$ \\
\hline $\begin{array}{l}\text { Chen et al. } \\
(2012)\end{array}$ & $\begin{array}{l}\text { Identificar los motivos de readmisión hospitalaria de } \\
4101 \text { pacientes con problemas cardiacos y, en espe- } \\
\text { cial, cómo se presentaba la transición entre el cuidado } \\
\text { del hospital y el de la comunidad. }\end{array}$ & $\begin{array}{l}24,7 \% \text { de los pacientes fueron readmitidos, debido a que los } \\
\text { planes de egreso varían sin tener una directriz claramente es- } \\
\text { tablecida. }\end{array}$ \\
\hline Foust (2007) & $\begin{array}{l}\text { Examinar cómo ocurren en la práctica los esfuerzos } \\
\text { de planeación del cuidado continuo. Observaron y en- } \\
\text { trevistaron a ocho enfermeras, mientras estas cuida- } \\
\text { ban a sus pacientes. }\end{array}$ & $\begin{array}{l}\text { Las expectativas que tienen las enfermeras del progreso de los } \\
\text { pacientes guían su planeación en el tiempo. En la práctica dia- } \\
\text { ria, la enseñanza al egreso es de carácter más específico que } \\
\text { institucional. }\end{array}$ \\
\hline $\begin{array}{l}\text { Chin-Yen et al. } \\
(2009)\end{array}$ & $\begin{array}{l}\text { Verificar el nivel de preparación del personal para } \\
\text { asumir un plan de egreso. }\end{array}$ & $\begin{array}{l}\text { Las enfermeras deben adquirir la competencia para adelantar } \\
\text { el plan de egreso. }\end{array}$ \\
\hline $\begin{array}{l}\text { Collier \& Har- } \\
\text { rington (2005) }\end{array}$ & $\begin{array}{l}\text { Determinar los actores del equipo de salud involucra- } \\
\text { dos y las variables que inciden en el plan de egreso en } \\
\text { hospitales de California. }\end{array}$ & $\begin{array}{l}\text { No se tienen en cuenta criterios de calidad sino la disponibi- } \\
\text { lidad de cupos y el recurso económico, lo cual, no coincide } \\
\text { con el conocimiento que tienen los responsables del plan de } \\
\text { egreso. Solamente el } 24 \% \text { del equipo de salud usó internet } \\
\text { para su decisión y en ello influyeron la falta de acceso a la tec- } \\
\text { nología y el rol limitado de algunos de los responsables. }\end{array}$ \\
\hline $\begin{array}{l}\text { Pichitporn- } \\
\text { chaia et al. } \\
(1999)\end{array}$ & $\begin{array}{l}\text { Revisar las prácticas de plan de egreso de las enfer- } \\
\text { meras tailandesas y cómo la transición hospital hogar } \\
\text { se incorpora como parte de la práctica. }\end{array}$ & $\begin{array}{l}\text { El plan de egreso es un proceso informal que se ve atravesado } \\
\text { por varios factores y debe ser cualificado. }\end{array}$ \\
\hline $\begin{array}{l}\text { Rhudy et al. } \\
\text { (2010) }\end{array}$ & $\begin{array}{l}\text { Revisar cómo toman las decisiones las enfermeras } \\
\text { con relación al plan de egreso de los pacientes y cómo } \\
\text { perciben su rol en este campo. }\end{array}$ & $\begin{array}{l}\text { La identificación de necesidades particulares se asocia a la ca- } \\
\text { pacidad de comunicación de las enfermeras y a la aplicación } \\
\text { de su propia competencia de tomar decisiones según la indi- } \\
\text { cación. }\end{array}$ \\
\hline $\begin{array}{l}\text { Watts et al. } \\
(2005)\end{array}$ & $\begin{array}{l}\text { Revisar cómo las enfermeras de cuidado crítico de- } \\
\text { finen el plan de egreso y su proceso de planeación y } \\
\text { logran, con base en ello, explicar la percepción sobre } \\
\text { los mismos. El estudio incluyó a } 218 \text { enfermeras de } \\
\text { cuidado crítico en Australia. }\end{array}$ & $\begin{array}{l}\text { Un número significativo de enfermeras reporta que no hay plan } \\
\text { de egreso y no saben qué debe contener. Quienes sí lo repor- } \\
\text { tan, evidencian tres temas comunes: 1) énfasis en el traslado } \\
\text { de los pacientes al servicio de hospitalización, 2) asegurar una } \\
\text { transición amable y armónica y 3) realizar la transición por req- } \\
\text { uerimiento de camas para otros pacientes. }\end{array}$ \\
\hline $\begin{array}{l}\text { Hanratty et al. } \\
(2012)\end{array}$ & $\begin{array}{l}\text { Explorar las experiencias de personas ancianas que } \\
\text { son trasladadas a sitios de cuidado al final de la vida. } \\
\text { Se abordaron } 30 \text { adultos, entre los } 69 \text { y } 93 \text { años, con } \\
\text { diagnóstico de enfermedad terminal y de diferentes } \\
\text { estratos socio-económicos. }\end{array}$ & $\begin{array}{l}\text { Se identifican cuatro categorías en su experiencia: 1) la priori- } \\
\text { zación del proceso de institucionalización; 2) el apoyo en los } \\
\text { diferentes sitios; } 3 \text { ) ser escuchados y, 4) la dignidad. A medida } \\
\text { que se trasladaron encontraron profesionales menos flexibles } \\
\text { y se sintieron menos escuchados. Es necesario concientizar a } \\
\text { los profesionales para buscar que la transición responda a las } \\
\text { necesidades del paciente. }\end{array}$ \\
\hline Krohn (2008) & $\begin{array}{l}\text { Identificar la importancia del plan de egreso en la } \\
\text { atención de pacientes ambulatorios sometidos a difer- } \\
\text { entes procedimientos diagnósticos. }\end{array}$ & $\begin{array}{l}\text { Las instrucciones de salida, su propósito, la definición de las } \\
\text { metas de estas instrucciones y planes, la consideración de los } \\
\text { obstáculos para comprender la instrucción, la necesidad de re- } \\
\text { visarla y la pertinencia de los impresos de las instrucciones de } \\
\text { salida, deben ser abordadas para generar un plan adecuado. }\end{array}$ \\
\hline $\begin{array}{l}\text { Han et al. } \\
(2009)\end{array}$ & $\begin{array}{l}\text { Reconocer el plan de egreso como un problema cen- } \\
\text { tral dentro del sistema de salud, en donde la estadía } \\
\text { hospitalaria tiende a ser más corta, los costos más al- } \\
\text { tos y, en consecuencia, se incrementa el énfasis en el } \\
\text { cuidado comunitario. }\end{array}$ & $\begin{array}{l}\text { En la medida en que los profesionales del área de la salud com- } \\
\text { prendan la importancia de dicho plan de egreso, se podrán dis- } \\
\text { minuir las readmisiones, los costos del servicio, el estrés para } \\
\text { los pacientes y mejorar la continuidad del cuidado. }\end{array}$ \\
\hline
\end{tabular}

Fuente: Construcción propia de las autoras. 2013. 
La categoría Instrumentos, incluyó la revisión de seis publicaciones que agrupa estudios que buscaron generar o probar instrumentos para apoyar la orientación o medición del plan de transición y de egreso hospitalario. Los instrumentos existentes orientan el plan de transición y el de egreso y se enfocan en características de integralidad, de seguridad y de continuidad. La revisión no reveló reporte de instrumentos en español y con pruebas psicométricas (Cuadro 2).

Cuadro 2. Instrumentos que se han utilizado para medir el plan de egreso hospitalario.

\begin{tabular}{|l|l|}
\hline \multicolumn{1}{|c|}{ Instrumento } & \multicolumn{1}{c}{ Descripción } \\
\hline Índice Generado en el Cliente. Annells et al. (2001) & $\begin{array}{l}\text { Valora y mide los resultados de la calidad de vida relacionados con el estado } \\
\text { de salud y que facilita el cuidado individualizado. Se revisó aplicabilidad y } \\
\text { utilidad de la herramienta por parte de enfermeras comunitarias en el esta- } \\
\text { blecimiento de planes de cuidado de enfermería y medición de resultados de } \\
\text { la calidad de vida del paciente. Aunque demostró ser una herramienta útil, no } \\
\text { reporta sus pruebas psicométricas. }\end{array}$
\end{tabular}

Cuestionario de egreso de Kenner. Kenner \& Boykova (2007)

Instrumento para medir la percepción del paciente sobre la calidad del cuidado de enfermería y servicios afines en un hospital de tercer nivel.

Senarath \& Gunawardena (2011)

Escala de Calidad de educación al dar de alta y escala de la Preparación para el alta hospitalaria. Bobay et al. (2010)

Instrumento de valoración de las necesidades de enfermería. Holland et al. (1998)

poulos et al. (2009)
Test estandarizado en idioma inglés, que demostró ser válido y confiable en la revisión de las experiencias de los padres con el cuidado y el soporte social. Consta de 38 ítems y se responde con valoración de tipo escala de Likert. Su aplicación permitió identificar las necesidades de información de los padres, sus roles, el nivel de estrés, el tipo de afrontamiento y los soportes o interacciones que requieren para asumir las responsabilidades de cuidado.

Esta herramienta surge de la experiencia de trabajo, la revisión de la literatura y la consulta con expertos. Para validarla se aplicó a 120 pacientes al momento del egreso. De los 72 ítems inicialmente propuestos, quedaron 43, que fueron calificados en ocho categorías que incluyen: aspectos interpersonales, eficiencia, competencia, confort, ambiente físico, limpieza, información personalizada, e instrucción general. El instrumento final de 36 ítems tuvo un alpha de Cronbach's de 0,91 y demostró ser válido para uso en hospitales.

Con 1982 pacientes que tenían ordenada su salida a la casa, se completó esta escala para determinar las diferencias en las percepciones de la calidad en la educación del egreso hospitalario y el grado de preparación para el alta hospitalaria y su relación con el reingreso a las urgencias.

Los hallazgos reflejaron una asociación entre el nivel de preparación para el alta hospitalaria y la edad en donde la herramienta resulta predictiva. Las herramientas mostraron ser válidas y confiables con todos los grupos etareos.

Surgió con base en la revisión de los requerimientos de las enfermeras de la comunidad y cuidado en casa para incluirlo en el plan de egreso hospitalario.

La herramienta contribuye en la comprensión de las necesidades de los pacientes entre el cuidado agudo y el crónico.

Es una herramienta que surgió para apoyar a las enfermeras en la revisión de una manera sencilla del cuidado de pacientes de ortopedia. Incluye aspectos de percepción de suministro de información, comunicación entre el hospital y las agencias de cuidado y continuidad en el cuidado.

Fuente: Construcción propia de las autoras. 2013.

En la categoría que referencia Características básicas del plan de transición y egreso y desarrollo teórico que lo respalda, se encontraron 24 publicaciones, cuya informa- ción, a pesar de ser heterogénea, reconoce la relevancia de la transición y el egreso como un momento de mayor riesgo para el cuidado seguro y continúo (Cuadro 3 ). 
Cuadro 3. Características básicas del plan de egreso.

\begin{tabular}{|c|c|}
\hline Autores & Características que debe tener el plan de egreso \\
\hline Hauser (2009) & $\begin{array}{l}\text { Comunicación clara y concisa, cuando se cambia de escenario a un paciente de cuidado paliativo, quien req- } \\
\text { uiere saber todos los detalles de su cuidado sin tener brechas en los detalles, para atender sus necesidades en } \\
\text { ese momento de transición de alta complejidad. }\end{array}$ \\
\hline Crocker et al. (2012) & Sistema de seguimiento continuo, a través de llamadas telefónicas, como parte integral del plan de egreso. \\
\hline Brand (2006) & $\begin{array}{l}\text { Contar con un equipo interdisciplinario con alta capacidad de toma de decisiones adecuada comunicación } \\
\text { de las enfermeras con los médicos. Enfermería asume un rol administrativo en la coordinación de programas } \\
\text { de egreso }\end{array}$ \\
\hline $\begin{array}{l}\text { Huber \& Mcclelland } \\
\text { (2003), }\end{array}$ & $\begin{array}{l}\text { Alta participación del paciente y su familia en el diseño del plan de egreso. Identificar las prioridades manifes- } \\
\text { tadas por los pacientes y sus cuidadores atendiendo a esas preferencias. }\end{array}$ \\
\hline Lowson et al. (2013) & $\begin{array}{l}\text { Vinculación activa de los cuidadores familiares, quienes hacen enormes contribuciones para mantener el } \\
\text { ritmo del buen cuidado durante la trayectoria de la enfermedad. Los cuidadores dedican gran esfuerzo para } \\
\text { maximizar el bienestar a través de identificar necesidades y abogar por los pacientes. }\end{array}$ \\
\hline Beltz (2013) & $\begin{array}{l}\text { Identificación de necesidades de cuidado en cada una de los servicios a donde se encuentra el paciente, } \\
\text { teniendo en cuenta la edad de los usuarios y las transiciones propias del paso de la niñez a la adultez. Los } \\
\text { adolescentes requieren, en su transición, recibir atención integral e interdisciplinaria y se debe atender esta } \\
\text { situación, para generar políticas públicas que respondan a las demandas de cuidado. }\end{array}$ \\
\hline $\begin{array}{l}\text { Mcbride \& Andrews } \\
(2012)\end{array}$ & $\begin{array}{l}\text { Implementar planes de fácil comprensión para los pacientes, incluyendo una capacitación para adquirir la } \\
\text { competencia de educación a la salida. }\end{array}$ \\
\hline Thraena et al. (2012) & $\begin{array}{l}\text { Transferir información integrada orientada al cumplimiento de metas, consolidar procesos de trabajo sis- } \\
\text { temático con retroalimentación y, mejorar la interdependencia del paciente empleando un Modelo de Sistema } \\
\text { Cognitivo Conjunto. }\end{array}$ \\
\hline Ok Im (2011) & $\begin{array}{l}\text { Utilización de marcos de referencia y teorías de rango medio relacionadas, como la de las Transiciones de } \\
\text { Meleis, la cual, es de gran aporte a la construcción teórica y avance dentro de la enfermería en la transición } \\
\text { hospital hogar del paciente. }\end{array}$ \\
\hline Bowles et al. (2003) & $\begin{array}{l}\text { Basarse en el modelo de déficit del auto cuidado de Orem, contar con instrumentos estandarizados, comu- } \\
\text { nicación electrónica, mayor educación y entrenamiento y personal creativo para superar los problemas de la } \\
\text { comunicación para garantizar la continuidad en el cuidado en el hogar. }\end{array}$ \\
\hline Bowman et al. (2012) & $\begin{array}{l}\text { Incluir en los planes de egreso a personas en fase de cuidado paliativo, la valoración de ocho dominios: la } \\
\text { estructura y el proceso, el físico, el psicológico y psiquiátrico, el social, el espiritual, religioso y existencial, el } \\
\text { cultural el cuidado de la muerte inminente y el ético legal. }\end{array}$ \\
\hline Santo \& Purden (2008) & $\begin{array}{l}\text { Utilización de herramientas informativas tales como cartillas y folletos para apoyar a las familias en la tran- } \\
\text { sición entre el hospital y la casa. }\end{array}$ \\
\hline Tellett et al. (2012) & $\begin{array}{l}\text { Los planes de egreso en cuidado paliativo deben contar con un sistema de acompañamiento a la familia en el } \\
\text { manejo de los síntomas del paciente en la etapa final. } \\
\text { Los criterios para transferir a un paciente en etapa terminal a su casa deben ser evaluados de manera diferen- } \\
\text { cial, dependiendo de las circunstancias y del posible apoyo. }\end{array}$ \\
\hline Oliver (2005) & $\begin{array}{l}\text { Consolidar modelos para abordar posibles eventos adversos en casa como las caídas y revisar si las interven- } \\
\text { ciones modifican los índices de estos eventos adversos. }\end{array}$ \\
\hline $\begin{array}{l}\text { Vargas \& Fernández } \\
\text { (2011) }\end{array}$ & $\begin{array}{l}\text { Construcción de modelos para la implementación de un Programa del Alta Hospitalaria Programada (PAH) } \\
\text { que incluya: una valoración de las necesidades del paciente, tanto al momento del ingreso como en el egreso; } \\
\text { coordinación con la red externa de salud y la reubicación de los usuarios con problemas sociales, así como } \\
\text { una agilización de los trámites administrativos. }\end{array}$ \\
\hline
\end{tabular}


Continuación Cuadro 3.

\begin{tabular}{|c|c|}
\hline Corbett et al. (2010) & $\begin{array}{l}\text { Mejorar el conocimiento del paciente y su familia sobre los medicamentos antes de salir. } \\
\text { Suministrar al paciente listas de medicamentos. } \\
\text { Realizar seguimiento luego del alta con visita domiciliaria o llamada telefónica. } \\
\text { Agendar visitas de seguimiento antes del alta. } \\
\text { Simplificar el régimen médico post hospitalario. }\end{array}$ \\
\hline $\begin{array}{l}\text { Hellesøa \& Lorensena } \\
\text { (2005) }\end{array}$ & $\begin{array}{l}\text { Es fundamental dar continuidad en el cuidado, reconociendo las necesidades del usuario que se requieren } \\
\text { para el manejo en casa. En tal sentido se afirma que es indispensable llegar a acuerdos sobre contenidos } \\
\text { mínimos que soporten el plan para los escenarios intra y extra hospitalarios. }\end{array}$ \\
\hline McDonald (2012) & $\begin{array}{l}\text { El plan de egreso se debe fundamentar en brindar un cuidado seguro durante la transición al hogar, con la } \\
\text { inclusión activa de los pacientes y sus familiares. } \\
\text { Son temas claves a tener en cuenta el manejo de las heridas y la administración de medicamentos. }\end{array}$ \\
\hline Hellesøa et al. (2004) & $\begin{array}{l}\text { Mantenimiento de comunicación activa y efectiva entre las enfermeras hospitalarias con las enfermeras que } \\
\text { trabajan en el hogar respecto a lo que ellas perciben como información significativa. Implementar registro } \\
\text { electrónico de estas afirmaciones. }\end{array}$ \\
\hline Jacob (1999) & $\begin{array}{l}\text { Se requiere coordinación de esfuerzos con las enfermeras de cuidado en el hogar para que se apoye a los } \\
\text { cuidadores buscando mayor conocimiento y habilidad en medio de esta transición. }\end{array}$ \\
\hline Weiss et al. (2008) & $\begin{array}{l}\text { Abordar los predictores de los padres para cuidar en el hogar a los niños que tienen salida hospitalaria tales } \\
\text { como las características de los padres, hijos y la hospitalización con la percepción de preparación para el } \\
\text { cuidado en casa; las prácticas de enfermería de educación al egreso y coordinación del cuidado con la prepar- } \\
\text { ación para el cuidado en casa de los padres al dar de alta; el alistamiento de los padres para salir del hospital } \\
\text { y, el alistamiento de los padres frente al cuidado en el hogar y la utilización del soporte después del alta. }\end{array}$ \\
\hline $\begin{array}{l}\text { Baker \& Wellman } \\
(2005)\end{array}$ & $\begin{array}{l}\text { Inclusión dentro del plan de egreso de temas relacionados con manejo de problemas nutricionales asociados } \\
\text { con la masticación, deglución, apetito, dietas cambiadas, la baja dentición. }\end{array}$ \\
\hline
\end{tabular}

Fuente. Construcción propia de las autoras. 2013

Con relación a Efectividad del plan de egreso para el cuidado seguro, integral y continúo, en esta categoría se exponen 11 estudios, que demuestran el nivel de efectividad del Plan de transición y de egreso hospitalario. Se muestra una tendencia que permitiría recomendar el plan de transición y de egreso hospitalario, para apoyar la continuidad, facilitar el cambio, precisar la directriz, evitar complicaciones y disminuir costo evitable (Cuadro 4).

Cuadro 4. Estudios que evidencian la efectividad del plan de egreso.

\begin{tabular}{|c|c|c|c|}
\hline Autores/año & Objetivo/Propósito & Método & Resultados \\
\hline
\end{tabular}


Continuación Cuadro 4.

\begin{tabular}{|c|c|c|c|}
\hline $\begin{array}{l}\text { Koelling et al. } \\
\text { (2005) }\end{array}$ & $\begin{array}{l}\text { Determinar el efecto de la educación del egreso } \\
\text { para pacientes con falla cardiaca. Seleccionaron } \\
\text { al azar } 223 \text { pacientes donde } 107 \text { de ellos, fueron } \\
\text { escogidos para recibir una educación de una } \\
\text { hora con una enfermera educadora. A todos los } \\
\text { pacientes se les hizo llamada a los } 30,90 \text { y } 180 \\
\text { días para saber sobre eventos clínicos, síntomas } \\
\text { y prácticas de auto cuidado. }\end{array}$ & $\begin{array}{l}\text { Ensayo clínico } \\
\text { aleatorizado } \\
\text { Longitudinal }\end{array}$ & $\begin{array}{l}\text { En los pacientes del grupo muestra se disminuyó } \\
\text { el número de días de hospitalización y la mortali- } \\
\text { dad, además de menor riesgo de complicación. }\end{array}$ \\
\hline $\begin{array}{l}\text { Roebuck } \\
(1999)\end{array}$ & $\begin{array}{l}\text { Explorar los niveles de ansiedad y depresión que } \\
\text { experimentaron los pacientes a la salida y cinco } \\
\text { semanas luego del alta y cómo el seguimiento } \\
\text { telefónico ayuda a mejorar esto. }\end{array}$ & $\begin{array}{l}\text { Descriptivo- Ex- } \\
\text { ploratorio } \\
\text { Longitudinal }\end{array}$ & $\begin{array}{l}\text { Se muestra una pequeña disminución en la } \\
\text { depresión durante el seguimiento }\end{array}$ \\
\hline $\begin{array}{l}\text { Ballard } \\
\text { Hernández } \\
(2010)\end{array}$ & $\begin{array}{l}\text { Implementar políticas de prácticas basadas en } \\
\text { la evidencia para educar a pacientes con falla } \\
\text { cardíaca. Los planes de educación estandari- } \\
\text { zados fueron creados y utilizados por las enfer- } \\
\text { meras. }\end{array}$ & $\begin{array}{l}\text { Descriptivo- } \\
\text { Exploratorio } \\
\text { Longitudinal }\end{array}$ & $\begin{array}{l}\text { Con el seguimiento telefónico se reforzó la edu- } \\
\text { cación, logrando bajar la readmisión de los pa- } \\
\text { cientes de } 35 \% \text { a } 18 \% \text {. }\end{array}$ \\
\hline $\begin{array}{l}\text { Pi-Chu et al. } \\
\text { (2005) }\end{array}$ & $\begin{array}{l}\text { Establecer un modelo de plan de egreso y eval- } \\
\text { uar su efectividad con pacientes de ortopedia. }\end{array}$ & $\begin{array}{l}\text { Descriptivo- } \\
\text { Piloto } \\
\text { Longitudinal }\end{array}$ & $\begin{array}{l}\text { El modelo resultó viable e incrementó la satisfac- } \\
\text { ción de los pacientes al momento del egreso pero } \\
\text { la prueba no contó con un grupo control para } \\
\text { poder valorar su efectividad real. }\end{array}$ \\
\hline $\begin{array}{l}\text { Gee et al. } \\
(2010)\end{array}$ & $\begin{array}{l}\text { Desarrollar un modelo de "reconciliación con la } \\
\text { medicación" denominado SERIOUS (solicitud, } \\
\text { examen, reconciliación, información, optimi- } \\
\text { zación, actualización y el compartir el régimen } \\
\text { de medicamentos) para respaldar a los pa- } \\
\text { cientes con falla cardiaca quienes tenían salida } \\
\text { reciente del hospital. Se programó una cita con } \\
\text { un farmaceuta y una enfermera clínica. }\end{array}$ & $\begin{array}{l}\text { Descriptivo- } \\
\text { Exploratorio } \\
\text { Longitudinal }\end{array}$ & $\begin{array}{l}\text { Se presentó un } 16 \% \text { de tasa de readmisión frente } \\
\text { al } 19,8 \% \text { en los demás pacientes. Se concluye que } \\
\text { los planes de egreso son un factor clave para pre- } \\
\text { venir reingresos. }\end{array}$ \\
\hline $\begin{array}{l}\text { Hellesøa et al. } \\
\text { (2005) }\end{array}$ & $\begin{array}{l}\text { Implementar un método electrónico de docu- } \\
\text { mentación integrada para los pacientes que } \\
\text { requieren cuidado continuo al egreso. } 287 \text { en- } \\
\text { fermeros hospitalarios y } 220 \text { de cuidado en casa } \\
\text { respondieron un cuestionario antes y después } \\
\text { del egreso de los usuarios. }\end{array}$ & $\begin{array}{l}\text { Descriptivo } \\
\text { Exploratorio } \\
\text { Transversal }\end{array}$ & $\begin{array}{l}\text { Con la implementación del registro electrónico } \\
\text { se disminuyeron las discrepancias entre las en- } \\
\text { fermeras hospitalarias y de cuidado en casa con } \\
\text { respecto al manejo de la información de los pa- } \\
\text { cientes. }\end{array}$ \\
\hline $\begin{array}{l}\text { Li-Chi et al. } \\
(2012)\end{array}$ & $\begin{array}{l}\text { Evaluar la efectividad de un programa de cuida- } \\
\text { do de enfermería que combinó planes de egreso } \\
\text { y seguimiento telefónico sobre el manejo del es- } \\
\text { trés y la carga del cuidador en pacientes con } \\
\text { falla cardiaca comparándolo con la atención } \\
\text { convencional. Se tomaron } 63 \text { pacientes en los } \\
\text { que las especialistas en tele enfermería dieron } \\
\text { asesoría } 24 \text { horas con monitoreo y seguimiento } \\
\text { telefónico. }\end{array}$ & $\begin{array}{l}\text { Cuasiexperi- } \\
\text { mental } \\
\text { Longitudinal }\end{array}$ & $\begin{array}{l}\text { Todos los indicadores mejoraron en el grupo } \\
\text { muestra por encima de los registros del grupo } \\
\text { control. }\end{array}$ \\
\hline Hiskett (2010) & $\begin{array}{l}\text { Explorar las implicaciones prácticas de la tera- } \\
\text { pia de presión negativa (TPN) en el cuidado en } \\
\text { casa para personas con heridas. Se estableció } \\
\text { una línea de base con foto y descripción. }\end{array}$ & $\begin{array}{l}\text { Descriptivo } \\
\text { Exploratorio } \\
\text { Longitudinal }\end{array}$ & $\begin{array}{l}\text { Se encontró un resultado clínico aceptable con un } \\
\text { costo menor y mayor comodidad de los pacientes. }\end{array}$ \\
\hline $\begin{array}{l}\text { Thrutchley et } \\
\text { al. (1997) }\end{array}$ & $\begin{array}{l}\text { Evaluaron un programa de cuidado en casa en } \\
\text { el cual entrenaron a las enfermeras para que } \\
\text { aplicaran en los pacientes una valoración inte- } \\
\text { gral y un sistema de monitoreo en casa junto } \\
\text { con una escala de seguridad y prevención de } \\
\text { nuevos eventos. }\end{array}$ & $\begin{array}{l}\text { Descriptivo } \\
\text { Exploratorio } \\
\text { Transversal }\end{array}$ & $\begin{array}{l}\text { Este tipo de programas facilita la transición del } \\
\text { hospital a la casa, disminuye longitud de la estadía } \\
\text { y sus costos mientras incrementa la satisfacción } \\
\text { del paciente. }\end{array}$ \\
\hline
\end{tabular}

Fuente. Construcción propia de las autoras, 2013. 
Vale la pena resaltar que se identifican múltiples vacios e investigación conceptual y metodológica, que se requieren de soporte para aplicar en la cualificación de la práctica en cuidado al paciente con enfermedad crónica. Atendiendo los parámetros propuestos por Arias \& Sánchez (2013), quienes señalan que el cuidado crónico debe cumplir con los atributos de acceso, seguridad, continuidad, fortalecimiento de la propia capacidad y uso de las tecnologías apropiadas para su soporte, esta revisión permite evidenciar la brecha entre la situación actual y la deseada, recordando que un sistema de salud que cuenta con madurez es aquel que atiende, de manera idónea, a las personas con enfermedad crónica (Samb et al. 2010).

Desde el punto de vista de enfermería, además de las consideraciones anteriores, es preciso señalar que el cuidado implica compromiso con el ser total y trasciende de realizar actividades a acompañar su experiencia de cuidado de la salud, lo cual, requiere abordar las transiciones y el egreso, como puntos críticos para los usuarios, dentro de la misma.

\section{CONCLUSIÓN}

Del análisis y de la reflexión acerca de los conceptos, las estrategias y los instrumentos encontrados en la literatura, se concluye que ella refleja, parcialmente, el vacío de la investigación frente a directrices concretas en el plan de transición y de egreso. A pesar que se reporta el desarrollo de algunos instrumentos que respaldan la valoración y la medición dentro del plan de egreso, no todos cuentan con pruebas psicométricas y ninguno de ellos es en español. Pocos documentos proponen características para el desarrollo teórico y son limitados los estudios que presentan los beneficios y la efectividad de este plan, para orientar el cuidado en diferentes poblaciones, señalando beneficios en reducción de costos, incremento de satisfacción y disminución de complicaciones innecesarias. La evidencia respalda la propuesta del plan de egreso para el cuidado integral, continuo y seguro, que requieren las personas con ECNT y sus cuidadores familiares e invita a continuar con este tipo de investigación, en respuesta a su inminente necesidad.

Conflicto de intereses: El manuscrito fue preparado y revisado con la participación de todos los autores, quienes declaramos que no existe ningún conflicto de intereses que ponga en riesgo la validez de los resultados presentados. Financiación: Dirección Nacional de Extensión, Universidad Nacional de Extensión. Primera Convocatoria Nacional de Extensión Solidaria. Programa Cuidando a cuidadores. 2011-2012.

\section{BIBLIOGRAFÍA}

1. ANNELLS, M.; KOCH, T.; BROWN, M. 2001. Client relevant care and quality of life: the trial of a Client Gene- rated Index (CGI) tool for community nursing. Int. J. Nursing Studies (EEUU). 38:9-16.

2. ARIAS, M.; SÁNCHEZ, B. 2013. Las cinco características necesarias para ofrecer cuidado institucional en situaciones de enfermedad crónica. Experiencias de formación e investigación para el cuidado de enfermería. Bogotá: Editorial Universidad Nacional de Colombia. En prensa.

3. BAKER, E.; WELLMAN, N. 2005. Nutrition concerns in discharge planning for older adults: A need for multidisciplinary collaboration. J. Am. Diet Assoc. (EEUU). 105:603-607.

4. BALLARD HERNÁNDEZ, J. 2010. Nurse practitioners improving the transition from hospital to home and reducing acute care readmission rates in heart failure patients. Heart \& Lung J. (EEUU). 39(4):365-367.

5. BARRERA, L.; PINTO, N.; SÁNCHEZ, B. 2006a. La habilidad de cuidado de los cuidadores familiares de personas con enfermedad crónica. Inv. Ed. Enfermería. (Colombia). 23(1):36-46.

6. BARRERA, L.; PINTO, N.; SÁNCHEZ, B.; GALVIS, C.; MORENO, M.; PINZÓN, M., ROMERO E. 2006b. La habilidad de cuidado de los cuidadores familiares de personas con enfermedad crónica: Un estudio comparativo en tres unidades académicas de enfermería. Inv. Ed. Enfermería. 24(1):36-46.

7. BEN MORDERCHAI, B.; HERMAN, A.; KERZMAN, H.; IRONY, A. 2010 Structured discharge education improves early outcome in orthopedic patients. Int. J. Orthoped. Trauma Nurs. (Reino Unido). 14(2):66-74.

8. BELTZ, C. 2013. Health care transition for adolescents with special healthcare needs: Where's nursing? Nurse Outlook. (EEUU). 61(5):258-265.

9. BOBAY, K.; JEROFKE, T.; WEISS, M.; YAKUSHEVA, O. 2010. Age-related differences in perception of quality of discharge teaching and readiness for hospital discharge. Geriat. Nurs. (EEUU). 31(3):178-187.

10. BOWLES, K.; FOUST, J.; NAYLOR, M. 2003. Hospital discharge referral decision making: a multidisciplinary perspective. Appl. Nurs. Res. (EEUU). 16(3):134-143.

11. BOWMAN, E.; SACHS, G.; EMMETT, T. 2012. Do hospitalto-home transitional care programs for older adults address palliative care domains? A systematic review. J. Pain Symptom. Manag. (EEUU). 43(2):420-421. 
12. BULL, M.; HANSEN, H.; GROSS, C. 2000. A professional-patient partnership model of discharge planning with elders hospitalized with heart failure. Appl. Nurs. Res. 13(1):19-28.

13. BRAND, S. 2006. Nurses' roles in discharge decision making in an adult high dependency unit. Intens. Crit. Care Nurs. (Reino Unido). 22:106-114.

14. BURNS, N.; GROVE, S.K. 2012. Investigación en Enfermería. Desarrollo de la práctica Enfermera basada en evidencia. Barcelona: Elsevier España. 614p.

15. COLLIER, E.; HARRINGTON, C. 2005. Discharge planning, nursing home placement, and the Internet. Nurse Outlook. 53:95-103.

16. CORBETT, C.; SETTER, S.; DARATHA, K.; NEUMILLER, J.; WOOD, L. 2010. Nurse identified hospital to home medication discrepancies: implications for improving transitional care. Geriat. Nurs. 31:188-196.

17. CHEN, J.; ROSS, J.S.; CARLSON, M.; LIN, Z.; NORMAND, S.T.; BERNHEIM, S.M.; DRYE, E.E.; LING, S.M.; HAN, L.F.; RAPP, M.T.; KRUMHOLZ, H.M. 2012. Skilled nursing facility referral and hospital readmission rates after heart failure or myocardial infarction. Am. J. Med. (EEUU). 125(1):100.e1-100.e9.

18. CHIN-YEN, H.; CHIN-YI, C.; HUI-LING, L. 2009. Another compulsory clinical competence for emergency nurses -Implementing effective discharge planning. Austral. Emerg. Nurs. J. 12(4):178-186.

19. CROCKER, J.; JEFFREY, L. 2012. Telephone followup as a primary care intervention for postdischarge outcomes improvement: A systematic review. Am. J. Med. 125:915-921.

20. FOUST, J.B. 2007. Discharge planning as part of daily nursing practice. Appl. Nurs. Res. 90:72-77.

21. FRISK, B. 2007. Safe transition to home: preparing the near-term infant for discharge. Newborn \& Infant Nurs. Rev. (EEUU). 7(2):106-113.

22. GEE, J.; LAFOREST, S.; PUGACZ, A.; GUTHRIE, A.; SALEM, W.; SABATKA, S.; ORTIZ, C.; HOOVER, D. 2010. Getting serious about transition: a medication reconciliation model of care in the management of heart failure. Heart \& Lung J. 39(4):366-375.
23. HADJISTAVROPOULOS, H.; GARRATT, S.; JANZEN, J.; BOURGAULT-FAGNOU, M.; SPICE, K. 2009. Development and evaluation of a continuity of care checklist for improving orthopedic patient discharge from hospital. Int. J. Orthoped. Trauma Nurs. 13:183-193.

24. HAN, C.; BARNARD, A.; CHAPMAN, H. 2009. Discharge planning in the emergency department: a comprehensive approach. J. Emerg Nurs. (EEUU). 35(6):525-528.

25. HANRATTY, B.; HOLMES, L.; LOWSON, E.; GRANDE, G.; ADDINGTON-HALL, J.; PAYNE, S. 2012. Older adults' experiences of transitions between care settings at the end of life in England: a qualitative interview study. J. Pain Symptom. Manag. 44(1):74-83.

26. HAUSER, J. 2009. Lost in transition: the ethics of the palliative care handoff. J. Pain Symptom. Manag. 37(1):930-933.

27. HELLESØA, R.; LORENSENA, M. 2005. Inter-organizational continuity of care and the electronic patient record: A concept development. Int. J. Nurs. Studies. 42:807-822.

28. HELLESØA, R.; SORENSENB, L.; LORENSENA, $M$. 2005. Nurses' information management across complex health care organizations. Int. J. Med. Informat. (EEUU). 74:960-972.

29. HELLESØA, R.; LORENSENA, M.; SORENSENB, L. 2004. Challenging the information gap - the patients transfer from hospital to home health care. Int. J. Nurs. Studies. 73:569-580.

30. HISKETT, G. 2010. Clinical and economic consequences of discharge from hospital with on-going TNP therapy: a pilot study. J. Tissue Viability. (Reino Unido). 19(1):16-21.

31. HOLLAND, D.; HANSEN, D.; MATT-HENSRUD, N.; SEVERSON, M.; WENNINGER, C. 1998. A nursing needs assessment instrument. Geriat. Nurs. 19:331334.

32. HUBER, D.; McCLELLAND, E. 2003. Patient preferences and discharge planning transitions. J. Prof. Nurs. (EEUU). 19:204-210.

33. OK IM, E. 2011. Transitions theory: A trajectory of theoretical development in nursing. Nurse Outlook. 59:278-285. 
34. JACOB, E. 1999. Making the transition from hospital to home: caring for the newly diagnosed child with cancer. Home Care Provid. (EEUU). 4(2):67-73.

35. KENNER, C.; BOYKOVA, M. 2007. Transition to home: family perspectives on care in Russia. Newborn \& Infant Nurs. Rev. 7(1):20-24.

36. KOELLING, T.; JOHNSON, M.; CODY, R.; AARONSON, K. 2005. Discharge education improves clinical outcomes in patients with chronic heart failure. J. Am. Heart Assoc. 111:179-185.

37. KROHN, D. 2008. Discharge instructions in the outpatient setting: nursing considerations. J. Radiol. Nurs. (EEUU). 27:29-33.

38. LI-CHI, CH.; WAN-CHOU, CH.; YU-TZU, D.; YI-LWUN, H. 2012. The effectiveness of telehealth care on caregiver burden, mastery of stress, and family function among family caregivers of heart failure patients: A quasi-experimental study. Int. J. Nurs. Studies. 49:1230-1242.

39. LIN, F.; CHABOYER, W.; WALLIS, M. 2009. A literature review of organizational, individual and teamwork factors contributing to the ICU discharge process. Austral. Crit. Care. 22:29-43.

40. LOWSON, E.; HANRATTY, B.; HOLMES, L.; ADDINGTON-HALL, J.; GRANDE, G.; PAYNE, S.; SEYMOUR, J. 2013. From 'conductor' to 'second fiddle': Older adult care recipients' perspectives on transitions in family caring at hospital admission. Int. J. Nurs. Studies. 50(9):1197-1205.

41. McBRIDE, M.; ANDREWS, G. 2012. The transition from acute care to home: a review of issues in discharge teaching and a framework for better practice. Canad. J. Cardiol. 28(Suppl 5):S436-S445.

42. McDONALD, S. 2012. Discharge readiness: an opportunity for change in the transition from hospital to home. Canad. J. Cardiol. 28(5):443-451.

43. OLIVER, D. 2005. Medical input, rehabilitation and discharge planning for patients with hip fracture: Why traditional models are not fit for purpose and how things are changing current. Anesth. \& Crit. Care. (Australia). 16:11-22.

44. ORGANIZACIÓN MUNDIAL DE LA SALUD. Estadísticas Sanitarias Mundiales 2013. Disponible desde internet en: http://apps.who.int/iris/bitstream/10665/82062/1/WHO_HIS_HSI_13.1_spa. pdf (con acceso 15/05/2013)

45. PI-CHU, L.; JIN-LIAN, W.; SU-YUCHANG, B.; FU-MEIYANG, C. 2005. Effectiveness of a discharge-planning pilot program for orthopedic patients in Taiwan. Int. J. Nurs. Studies. 42:723-731.

46. PICHITPORNCHAIA, W.; ANNETTE, S.; TASSANA, B. 1999. Discharge planning and transitional care: issues in Thai nursing. Int. J. Nurs. Studies. 36:355362.

47. PINTO, N.; BARRERA, L.; SÁNCHEZ, B.; FIGUEROA, P.; BLANCO DE CAMARGO, L. 2006. Habilidad de cuidado de los cuidadores familiares de personas con enfermedad crónica. Mirada Internacional. Aquichán. (Colombia). 6(1):22-33.

48. RHUDY, L.; HOLLAND, D.; BOWLES, K. 2010. Iluminating hospital discharge planning: staff nurse decision making. Appl. Nurs. Res. 23(4):198-206.

49. ROEBUCK, A. 1999. Telephone support in the early post discharge period following elective cardiac surgery: does it reduce anxiety and depression levels? Intens. Crit. Care Nurs. 15:142-146.

50. SAMB, B.; DESAI, N.; NISHTAR, S.; MENDIS, S.; BEKEMAN, H.; WRIGHT, A. 2010. Prevention and management of chronic disease: a litmus test for health systems strengthening in low-income and middle-income countries. The Lancet. 376(9754):1785-1797.

51. SÁNCHEZ, B. 2011. Cuidado seguro: la nueva tendencia en el cuidado de la salud. Actual. Enferm. (Colombia). 14(2):27-32.

52. SANTO, A.; PURDEN, M. 2008. Developing an information booklet for parents and caregivers of children recovering from spinal fusion surgery. Int. J. Orthoped. Trauma Nurs. 12:84-89.

53. SENARATH, U.; GUNAWARDENA, N. 2011. Development of an instrument to measure patient perception of the quality of nursing care and related hospital services at the National Hospital of Sri Lanka. Asian Nurs. Res. (Corea). 5(2):71-80.

54. TELLETT, L.; PYLE, L.; COOMBS, M. 2012. End of life in intensive care: Is transfer home an alternative? Intens. Crit. Care Nurs. (Reino Unido). 28:234-241. 
55. THRAENA, I.; BAIRB, B.; MULLINC, S.; WEIR, C. 2012. Characterizing "information transfer" by using a joint cognitive systems model to improve continuity of care in the aged. Int. J. Med. Informat. 81:435-441.

56. THRUTCHLEY, D.; DELONG, D.; RYMER, M. 1997. Stroke home health care: The transition to independence. J. Stroke Cerebrovasc. Dis. (EEUU). 6(6):465-471.

57. VARGAS, Z.; FERNÁNDEZ, R. 2011. Programa de alta hospitalaria programada para preparar el egreso del paciente y familia. Rev. Enferm. Actual en Costa Rica. 21:1-20.
58. WATTS, R.; PIERSON, J.; GARDNER, H. 2005. How do critical care nurses define the discharge planning process? Intens. Crit. Care Nurs. 21:39-46.

59.WEISS, M.; JOHNSON, N.; MALIN, S.; JEROFKE, T.; LANG, C.; SHERBURNE, E. 2008. Readiness for discharge in parents of hospitalized children. J. Ped. Nurs. (EEUU). 23(4):282-295.

Recibido: Julio 24 de 2013

Aceptado: Diciembre 5 de 2013

\section{Como citar:}

Sánchez, B.; Carrillo, G.M.; Barrera, L. 2014. El plan de transición y egreso hospitalario y su efecto en el cuidado de la salud: una revisión integrada. Rev. U.D.C.A Act. \& Div. Cient. 17(1): 13-23. 
